\section{Molecular diagnostics useful in autosomal dominant polycystic kidney disease}

Most cases of autosomal dominant polycystic kidney disease (ADPKD) arise as a result of mutations in the $P K D 1$ or $P K D 2$ genes. More than 200 PKD1 mutations and more than 50 $P K D 2$ mutations have been reported in ADPKD to date. To determine the utility of gene-based mutation screening for diagnosis of ADPKD, Zhao et al. examined genomic DNA from five individuals for pathologic PKD1 and PKD2 mutations, by use of a commercial molecular diagnostic service. Three of the five patients had been referred for assessment of renal cystic disease and had no family history of ADPKD; the remaining two belonged to families with a history of ADPKD and were being evaluated as living-related kidney donors.

In the individuals without a family history of ADPKD, gene-based mutation screening provided unequivocal evidence of de novo pathogenic mutation in PKD1 in one case and strong evidence of de novo pathogenic mutation in PKD2 in another. In the third individual, genetic analysis identified an unclassified variant of $P K D 1$, the clinical significance of which is unclear. In the two individuals being evaluated as living-related kidney donors, gene-based mutation screening cleared one as a donor but was indeterminate in the other because unclassified $P K D 1$ variants were identified. DNA linkage studies in the family of this second individual provided strong evidence that ADPKD was not present in this case.

The authors conclude that use of molecular diagnostics should be considered if the diagnosis of ADPKD is uncertain. When gene-based mutation screening proves indeterminate, DNA linkage analysis can be useful in families with a number of affected individuals.

Original article Zhao X et al. (2008) Molecular diagnostics in autosomal dominant polycystic kidney disease: utility and limitations. Clin J Am Soc Nephrol 3: 146-152

\section{Losartan is renoprotective in diabetic nephropathy despite causing hemoglobin level to drop}

Mohanram et al. performed a post hoc analysis of the RENAAL study to investigate whether use of the angiotensin-receptor blocker losartan is associated with a reduction in hemoglobin level, and whether such a decline affects losartan's renoprotective properties.

RENAAL was a randomized, double-blind, placebo-controlled study that investigated whether long-term losartan use slowed progression of kidney disease among 1,513 patients with type 2 diabetes and overt nephropathy.

The baseline mean hemoglobin level was $125 \mathrm{~g} / \mathrm{l}(12.5 \mathrm{~g} / \mathrm{dl})$ in both the losartan and the placebo group. Over the first year of the study, the mean hemoglobin level dropped more rapidly in the losartan group than in the placebo group $(119 \mathrm{~g} / \mathrm{l}[11.9 \mathrm{~g} / \mathrm{dl}]$ vs $123 \mathrm{~g} / \mathrm{l}$ $[12.3 \mathrm{~g} / \mathrm{dl}]$ at year 1$)$. After the initial drop, the hemoglobin level stabilized in the losartan group but continued to decline gradually in the placebo group $(119 \mathrm{~g} / \mathrm{l}[11.9 \mathrm{~g} / \mathrm{dl}]$ and $118 \mathrm{~g} / \mathrm{l}$ $[11.8 \mathrm{~g} / \mathrm{dl}]$, respectively, at year 4).

The severity of proteinuria decreased in both groups every year over 4 years of follow-up, but the percentage decrease each year was greater in losartan-treated patients $(P<0.001)$. Losartan was associated with a significantly reduced risk of end-stage renal disease (ESRD) compared with placebo (relative risk reduction $35.8 \%$ for patients with baseline hemoglobin $<120 \mathrm{~g} /[$ [12.0 g/dl], $P=0.004$, and $30.6 \%$ for those with baseline hemoglobin $\geq 120 \mathrm{~g} / \mathrm{l}, P=0.026$ ). Among patients with a baseline hemoglobin level $<120 \mathrm{~g} / \mathrm{l}$, losartan significantly reduced the risk of ESRD or death in comparison with placebo (relative risk reduction $28.6 \%$; $P=0.006$ ).

The authors conclude that, despite a decline in hemoglobin level, losartan maintained its beneficial effects on proteinuria, ESRD, and a combined end point of ESRD or death, in patients with diabetic nephropathy.

Original article Mohanram A et al. (2008) The effect of losartan on hemoglobin concentration and renal outcome in diabetic nephropathy of type 2 diabetes. Kidney Int 73: 630-636

\section{Standardized terminology and diagnostic criteria for wasting syndromes in kidney disease}

An expert panel convened by the International Society of Renal Nutrition and Metabolism has delivered recommendations for the standardization of definitions concerning wasting, malnutrition and inflammation syndromes in 